\title{
Hybrid performance modelling of opportunistic networks
}

\author{
Luca Bortolussi $^{1} \quad$ Vashti Galpin ${ }^{2} \quad$ Jane Hillston $^{2}$ \\ ${ }^{1}$ Department of Mathematics and Computer Science, University of Trieste \\ ${ }^{2}$ Laboratory for Foundations of Computer Science, School of Informatics, University of Edinburgh \\ luca@dmi.units.it, Vashti.Galpin@ed.ac.uk, Jane.Hillston@ed.ac.uk
}

\begin{abstract}
We demonstrate the modelling of opportunistic networks using the process algebra stochastic HYPE. Network traffic is modelled as continuous flows, contact between nodes in the network is modelled stochastically, and instantaneous decisions are modelled as discrete events. Our model describes a network of stationary video sensors with a mobile ferry which collects data from the sensors and delivers it to the base station. We consider different mobility models and different buffer sizes for the ferries. This case study illustrates the flexibility and expressive power of stochastic HYPE. We also discuss the software that enables us to describe stochastic HYPE models and simulate them.
\end{abstract}

\section{Introduction}

Hybrid behaviour can arise in widely varying different contexts, both engineered and natural, pure and abstracted. Such systems have elements which are subject to continuous change, interleaved with discrete events which may change the elements themselves as well as their mode of evolution. The continuous aspect of the behaviour may be pure in that it is a physical entity which has continuous values, such as temperature or pressure, or may be abstracted as an approximation of a discrete quantity such as concentrations of biochemical species within a cell. Thus examples of hybrid systems include thermostatically controlled heating systems and genetic regulatory networks, such as the repressilator [13, 18].

In this paper we consider an engineered system with abstract continuity: an opportunistic network [22, 32]. In such a network, nodes experience periods of disconnectedness, during which they nevertheless may accumulate traffic in the form of packets. Sporadic connectivity is provided by occasional proximity of other nodes. Such connectivity is then exploited to further the progress of packets towards their destination (hence the term opportunistic). There are many interesting questions about performance and capacity planning for such networks, but a detailed discrete state representation in which all packets are treated individually can rapidly exceed feasible analysis and can also be expensive in terms of time. Instead, here, we abstract the traffic to be a fluid quantity rather than discrete packets and model the system as a stochastic hybrid system.

Process algebras have a long-established history of use for compositional modelling and analysis of systems with concurrent behaviour. Moreover, when extended with stochastic variables to represent duration and relative probability of events, they have been successfully applied to performance modelling and other forms of quantified analysis. HYPE is one of several recently defined process algebras which extend this capability to the modelling of hybrid systems [28, 17]. In HYPE the focus is on a finegrained compositionality, in which all the influences or flows which impact the continuous variables in the system are modelled explicitly. Importantly, addressing the modelling in this style removes the need to include ordinary differential equations (ODEs) governing the continuous evolution of such variables in the syntax of the model. Instead the dynamic behaviour emerges, via the semantics of the language, when the components are composed. Moreover, this fine-grained approach gives the language more expressiveness, than say, hybrid automata, as recently demonstrated in [16]. Furthermore, the use of

M. Massink and H. Wiklicky (Eds.):Workshop on Quantitative Aspects of Programming Languages and Systems 2012 (QAPL 2012)

EPTCS 85, 2012, pp. 106-121 doi 10.4204/EPTCS.85.8 (c) L. Bortolussi, V. Galpin \& J. Hillston

This work is licensed under the Creative Commons Attribution License. 
flows as the basic elements of model construction has advantages such as ease and simplification of modelling. This approach assists the modeller by allowing them to identify smaller or local descriptions of the model and then to combine these descriptions to obtain the larger system.

In the original definition of HYPE, discrete actions were termed events and these were always considered to be instantaneous although they could be subject to an activation condition determining just when the instantaneous jump would occur. A distinction was made between urgent and non-urgent events. Most activation conditions are expressed in terms of conditions on the evolving values of continuous variables and urgent events are triggered immediately when such conditions become true. In contrast, non-urgent events were not tied to the continuous state of the system (denoted by the undefined activation condition $\perp$ ) and could occur randomly at some unspecified time in the future. A recent extension of HYPE [7] refined this notion of non-urgent events, by introducing stochastic events. These events have an activation condition that is a random variable, capturing the probability distribution of the time until the event occurs. Thus these events still occur non-deterministically, but they are now quantified and so the models admit quantitative analysis. In order to carried out this type of analysis, a novel software tool has been developed which can simulate stochastic HYPE models.

This extended HYPE is ideal for modelling opportunistic networks in which we wish to study the emergent properties when nodes establish contact intermittently, but according to some probability distribution. In other words, we have some expectation of the frequency with which connections are formed, rather than admitting the possibility that this can be indefinitely postponed, as would be the case with non-urgent events. This is more realistic since the intermittent connectivity is usually provided by nodes embedded in vehicles which make regular visits.

The case study presented in this paper consists of stationary nodes that record multimedia data, specifically video, and a mobile node on a vehicle that collects the data and delivers it to a stationary base station. Since a characteristic of multimedia is very high data volume, this scenario is particularly appropriate for the fluid approach that we take here. We are specifically interested in two parameters of the model: how much data storage is required for the ferry and how often should it interact with the video sensors.

The rest of this paper is organised as follows. In Section 2 we briefly recall the basic notions of stochastic HYPE by means of a running example. This includes an account of the novel software tool which has been developed to simulate stochastic HYPE models. Next, in Section 2.1 we give a more detailed description of opportunistic networks, and the particular system we are considering. Section 4 presents a general framework for describing opportunistic networks, presents the stochastic HYPE model of the case study and the results of its analysis. Finally, Sections 5 and 6 discuss related work, future research and draw final conclusions.

\section{Stochastic HYPE}

In this section we present the definition of stochastic HYPE [7] and introduce a small example to illustrate the definition. More details about the language can be found in [7, 17, 16]. We consider a basic model of a network node with a buffer, which can receive packets from an input channel and send packets to an output channel. We assume that the number of packets that travel through the node and that are stored in the buffer is large, hence we describe them as a fluid quantity. Received packets are stored in the buffer, waiting to be sent. We allow reception and sending of packets to happen concurrently, but it is equally simple to enforce a mutually exclusive send/receive policy. We also assume that uplinks and downlinks are not always working, but they are activated and deactivated depending on the availability 


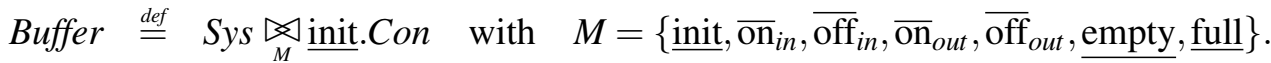

$$
\begin{aligned}
& \text { Sys } \stackrel{\text { def }}{=} \text { Input } \underset{\{\text { init }\}}{\bigotimes} \text { Output } \\
& \text { Input } \stackrel{\text { def }}{=} \overline{\mathrm{on}}_{i n}:\left(\text { in }, r_{\text {in }}, \text { const }\right) . \text { Input }+\overline{\mathrm{off}}_{i n}:(\text { in }, 0, \text { const }) . \text { Input }+ \\
& \text { full:(in, 0, const).Input }+ \text { init:(in, } 0, \text { const).Input } \\
& \text { Output } \stackrel{\text { def }}{=} \overline{\mathrm{on}}_{\text {out }}:\left(\text { out },-r_{\text {out }}, \text { const }\right) . \text { Output }+\overline{\text { off }}_{\text {out }}:(\text { out }, 0, \text { const }) . \text { Output }+ \\
& \text { empty: (out, } 0 \text {, const }) \text {.Output }+\underline{\text { init: }} \text { (out }, 0, \text { const }) \text {.Output }
\end{aligned}
$$

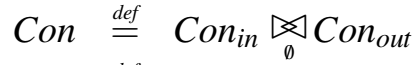

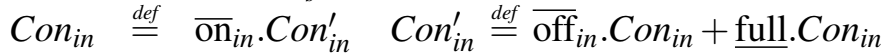

$$
\begin{aligned}
& \text { Con }_{\text {out }} \stackrel{\text { def }}{=} \overline{\mathrm{on}}_{\text {out }} . \text { Con }_{\text {out }}^{\prime} \quad \text { Con }_{\text {out }}^{\prime} \stackrel{\text { def }}{=} \overline{\mathrm{off}}_{\text {out }} \cdot \text { Con }_{\text {out }}+\text { empty. } \text { Con }_{\text {out }} \\
& \begin{array}{ll}
i v(\text { in })=B & i v(\text { out })=B
\end{array} \\
& e c(\underline{\text { init }})=\left(\text { true }, B^{\prime}=b_{0}\right) \\
& e c\left(\overline{\mathrm{on}}_{\text {in }}\right)=\left(k_{\text {in }}^{\text {on }}, \text { true }\right) \quad e c\left(\overline{\mathrm{off}}_{\text {in }}\right)=\left(k_{\text {in }}^{\text {off }}, \text { true }\right) \\
& e c\left(\overline{\mathrm{on}}_{\text {out }}\right)=\left(k_{\text {out }}^{\text {on }}, \text { true }\right) \quad e c\left(\overline{\text { off }}_{\text {out }}\right)=\left(k_{\text {out }}^{\text {off }}, \text { true }\right) \\
& e c(\underline{\text { full }})=\left(B=\max _{B}, \text { true }\right) \quad e c(\underline{\text { empty }})=(B=0, \text { true })
\end{aligned}
$$

Figure 1: Simple network node model in stochastic HYPE.

of a connection. These events are described as stochastic, with firing times governed by exponential distributions. Finally, incoming traffic has to be stopped if the buffer becomes full and outgoing traffic has to be stopped when the buffer is empty.

HYPE modelling is centred around the notion of flow, which is intended here as some sort of influence continuously modifying one variable. Both the strength and form of a flow can be changed by events. In our example, there are two flows modifying the buffer level, modelled by the continuous variable $B$, namely reception and sending of packets. Flows are described by the uncontrolled system, a composition of several sequential subcomponents, each modelling how a specific flow is changed by events. For instance, in Figure 1, the subcomponent Input describes the inflow of packets in the buffer. This subcomponent reacts to four events: $\overline{\mathrm{on}}_{i n}$ and $\overline{\mathrm{off}}_{i n}$, modelling the activation and deactivation of the uplink; full, modelling the suspension of incoming traffic due to the buffer becoming full; and init, the first event that sets the initial value of the influence. The tuple (in, $r_{i n}$, const) following event $\overline{\mathrm{on}}_{i n}$, is called an activity or an influence and describes how the input affects the buffer level when it is in effect: in is the name of the influence, which provides a link to the target variable of the flow ( $B$ in our example), $r_{i n}$ is the strength of the influence and const is the influence type, identifying the functional form of the flow (which is specified separately by the interpretation $\llbracket$ const $\rrbracket=1$ ). When the input is switched off, the influence $\left(\right.$ in,$r_{i n}$, const $)$ is replaced by (in, 0 , const) i.e. the influence strength of the input becomes zero. The other subcomponent affecting buffer level is the output component, modelling the sending of packets. States of a HYPE model are collections of influences, one for each influence name, defining a set of ordinary differential equations describing the continuous evolution of the system. For instance, (in, $r_{i n}$, const $)$ contributes to the ODE of $B$ with the summand $r_{i n} \llbracket$ const $\rrbracket=r_{i n}$. 
The controller Con, instead, is used to impose causality on events, reflecting natural constraints or design choices. For instance, $\mathrm{Con}_{\text {in }}$ models the fact that the reception of packets can be turned off only after being turned on. Furthemore, it describes termination of the input if the buffer becomes full, but only if the uplink is active.

Events in stochastic HYPE are of two kinds, either stochastic or deterministic. Deterministic events $\underline{\mathrm{a}} \in \mathscr{E}_{d}$ happen when certain conditions are met by the system. These event conditions are specified by a function $e c$, assigning to each event a guard or activation condition (a boolean predicate depending on system variable, stating when a transition can fire) and a reset (specifying how variables are modified by the event). For example, $e c(\underline{\text { full }})=\left(B=\max _{B}\right.$, true $)$ states that the uplink is shut down when the buffer reaches its maximum capacity $\max _{B}$, and no variable is modified. If we wanted to model a policy throwing away a fraction $\rho$ of the packets when the buffer becomes full, then we could have defined $e c(\underline{\text { full }})=\left(B=\max _{B}, B^{\prime}=(1-\rho) B\right)$. Deterministic events in HYPE are urgent, meaning that they fire as soon as their guard becomes true.

Stochastic events $\overline{\mathrm{a}} \in \mathscr{E}_{S}$ have an event condition composed of a stochastic rate (replacing the guard of deterministic events) and a reset. For instance, $e c\left(\overline{\mathrm{on}_{\text {in }}}\right)=\left(k_{i n}^{o n}\right.$, true $)$ states that the reception of packets is a stochastic event happening at times exponentially distributed with constant rate $k_{i n}^{o n}$. In general, rates define exponential distributions and can be functions of the variables of the system.

For completeness, we provide the formal definition of the syntax of stochastic HYPE.

Definition 1 A stochastic HYPE model is a tuple (ConSys, $\left.\mathscr{V}, I N, I T, \mathscr{E}_{d}, \mathscr{E}_{s}, \mathscr{A}, e c, i v, E C, I D\right)$ where

- ConSys is a controlled system as defined below.

- $\mathscr{V}$ is a finite set of variables.

- IN is a set of influence names and IT is a set of influence type names.

- $\mathscr{E}_{d}$ is the set of instantaneous events of the form $\underline{\mathrm{a}}$ and $\underline{\mathrm{a}}_{i}$.

- $\mathscr{E}_{s}$ is the set of stochastic events of the form $\overline{\mathrm{a}}$ and $\overline{\mathrm{a}}_{i}$.

- $\mathscr{A}$ is a set of activities of the form $\alpha(\mathscr{W})=(\imath, r, I(\mathscr{W})) \in(I N \times \mathbb{R} \times I T)$ where $\mathscr{W} \subseteq \mathscr{V}$.

- ec: $\mathscr{E} \rightarrow$ EC maps events to event conditions. Event conditions are pairs of activation conditions and resets. Resets are formulae with free variables in $\mathscr{V} \cup \mathscr{V}^{\prime}$. Activation conditions for instantaneous events $\mathscr{E}_{d}$ are formulas with free variables in $\mathscr{V}$, while for stochastic events of $\mathscr{E}_{s}$, they are functions $f: \mathbb{R}^{|\mathscr{V}|} \rightarrow \mathbb{R}^{+}$.

- iv $: I N \rightarrow \mathscr{V}$ maps influence names to variable names.

- EC is a set of event conditions.

- ID is a collection of definitions consisting of a real-valued function for each influence type name $\llbracket I(\mathscr{W}) \rrbracket=f(\mathscr{W})$ where the variables in $\mathscr{W}$ are from $\mathscr{V}$.

- $\mathscr{E}, \mathscr{A}$, IN and IT are pairwise disjoint.

Definition 2 A controlled system is constructed as follows.

- Subcomponents are defined by $C_{S}(\mathscr{W})=S$, where $C_{s}$ is the subcomponent name and $S$ satisfies the grammar $S^{\prime}::=\mathrm{a}: \alpha . C_{s} \mid S^{\prime}+S^{\prime}\left(\mathrm{a} \in \mathscr{E}=\mathscr{E}_{d} \cup \mathscr{E}_{S}, \alpha \in \mathscr{A}\right)$, with the free variables of $S$ in $\mathscr{W}$.

- Components are defined by $C(\mathscr{W})=P$, where $C$ is the component name and $P$ satisfies the gram$\operatorname{mar} P^{\prime}::=C_{s}(\mathscr{W})|C(\mathscr{W})| P^{\prime} \underset{L}{\bowtie} P^{\prime}$, with the free variables of $P$ in $\mathscr{W}$ and $L \subseteq \mathscr{E}$. 
- An uncontrolled system $\Sigma$ is defined according to the grammar $\Sigma^{\prime}::=C_{S}(\mathscr{W})|C(\mathscr{W})| \Sigma^{\prime} \underset{L}{\bowtie} \Sigma^{\prime}$, where $L \subseteq \mathscr{E}$ and $\mathscr{W} \subseteq \mathscr{V}$.

- Controllers only have events: $M::=\underline{\text { a. }} M|0| M+M$ with $\underline{\mathrm{a}} \in \mathscr{E}$ and $L \subseteq \mathscr{E}$ and Con $::=$ $M \mid$ Con $\underset{L}{ }$ Con.

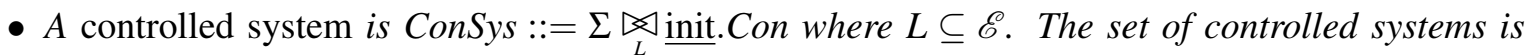
$\mathscr{C}_{\text {Sys. }}$

The semantics of HYPE has been defined in [17, 16], where a mapping to Hybrid Automata [20] is also discussed. The semantics of stochastic HYPE [7], instead, is given in terms of TDSHA (TransitionDriven Stochastic Hybrid Automata, [8]), which are a high level representation of PDMPs (Piecewise Deterministic Markov Processes, [11]). PDMPs are stochastic hybrid processes which interleave a deterministic evolution, described by a set of differential equations (depending on the current discrete mode of the system), with discrete jumps, which can be of two types: spontaneous, happening at exponentially distributed random times, and forced, happening when specific conditions on system variables are met. Both kind of events can reset the state of the system according to a specified reset policy. Intuitively, the dynamics of a stochastic HYPE model is as follows: the system variables will evolve following the solution of a set of ODE, defined by the influences active in the system, one for each influence name. The events that can happen, instead, are determined by the current state of the controller. Active stochastic events happen at random times, while deterministic events happen when their guard becomes true. In both cases, the reset policy of the event is applied. Moreover, the state of the controller and the set of active influences is updated according to model structure. In particular, all influences preceded in subcomponents by the event that occurred will replace the ones with the corresponding name, so that the continuous dynamics can have different modes of operation.

All HYPE models that will be considered in the paper comply with the definition of well-defined HYPE models, given in [16]. Essentially, each subcomponent must be a self-looping agent of the form $S=\sum_{i=1}^{k} \mathrm{a}_{i}: \alpha_{i} . S+$ init: $\alpha . S$, with each $\alpha_{i}$ of the form $\left(i_{S}, r_{i}, I_{i}\right)$, where $i_{S}$ is an influence name appearing only in subcomponent $S$. Furthermore, synchronization must involve all shared events.

\subsection{Simulation software}

In this section, we provide some details about the implementation of stochastic HYPE that we used to analyse the model of an opportunistic network presented in the paper. This software tool supports an automatic extraction of basic statistics from a set of stochastic runs, and has plotting facilities (including 3D plots and distribution histograms) and data export facilities. Furthermore, it supports automatic exploration of the parameter space. The user interface is command-line-based, and uses a simple script language to instruct the software.

The basic idea behind the implementation, written in Java and available on request from the authors, is to flatten a HYPE model into a representation in which additional discrete variables (i.e., variables that can take only a finite set of values) are introduced to keep track of the current active influences and the current states of the controller. The number of variables required for this encoding is easily seen to be linear in the size of the system, as it requires an additional number of variables equal to the number of different subcomponents plus the number of different states of the controller. Furthermore, this approach has the advantage of avoiding an explicit construction of all the modes of the (stochastic) hybrid automaton associated with a HYPE model. This is possible since modes of such an automaton are uniquely identified by the values of the discrete variables introduced. 
hype model network_node

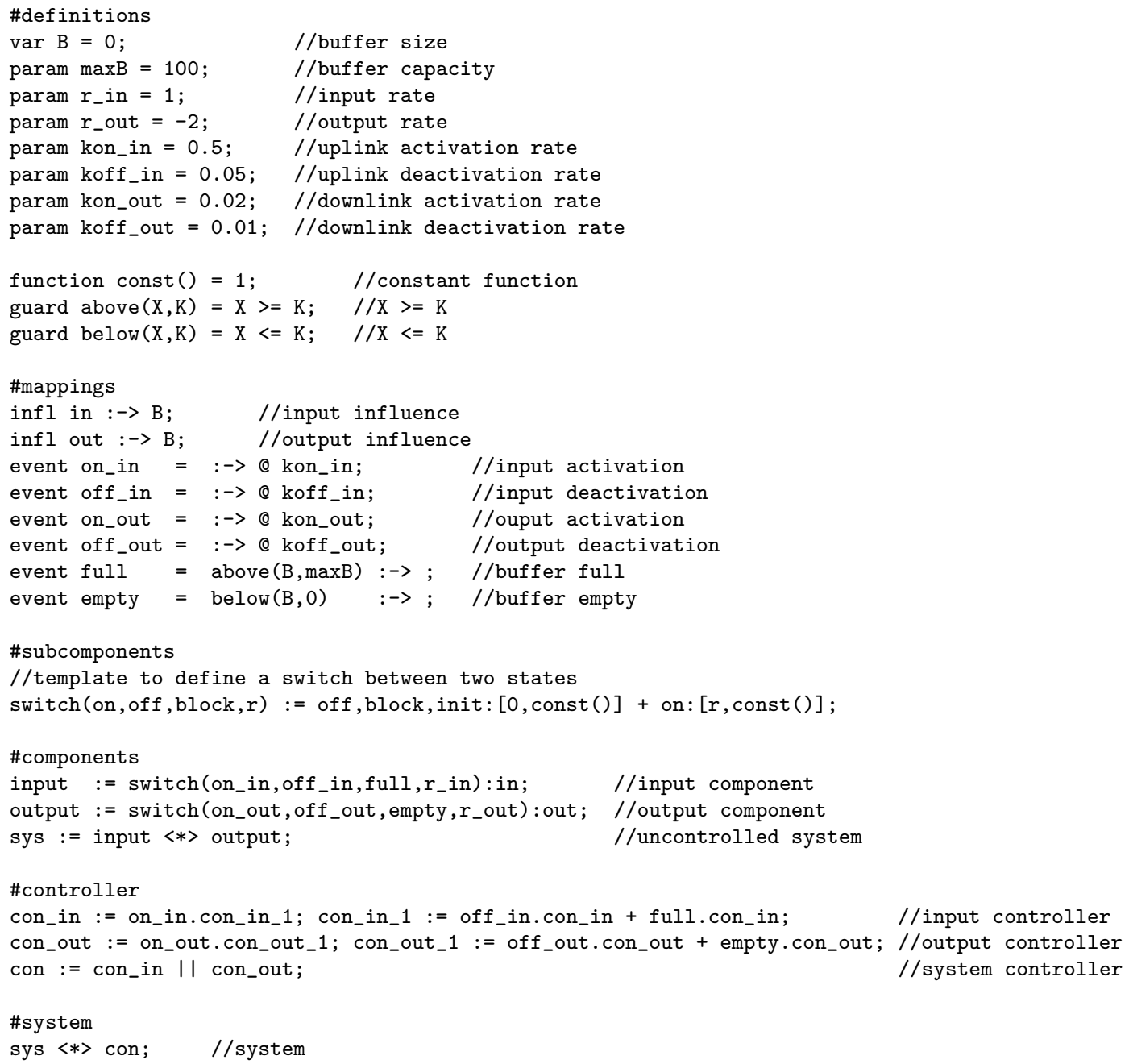

Figure 2: Code for the example given in Figure 1.

To illustrate how the encoding works in terms of the generated ODEs, consider the Input component of Section 2. To model which influence is active between $\alpha_{1}=\left(\right.$ in,$r_{i n}$, const $)$ and $\alpha_{0}=($ in, 0, const $)$, we need a new variable, $I_{\text {Input }}$, taking values in $\{0,1\}$. If $I_{\text {Input }}$ equals zero, the active influence is $\alpha_{0}$, otherwise the active influence is $\alpha_{1}$. This means that the component of the ODEs associated with variable $B$ generated by Input is of the form $r_{\text {in }} \llbracket$ const $\rrbracket\left\langle I_{\text {Input }}=1\right\rangle+0 \llbracket$ const $\rrbracket\left\langle I_{\text {Input }}=0\right\rangle=r_{\text {in }}\left\langle I_{\text {Input }}=1\right\rangle$, where $\langle\cdot\rangle$ denotes the logical value of a boolean predicate expressed as 0 or 1 . Resets and guards of events are modified in order to correctly update the discrete variables introduced. For instance, the reset of event full resets $I_{\text {Input }}$ to 0 .

The tool provides simulation of stochastic and non-stochastic HYPE models, and uses the Java mathematical library MathCommons [1] to numerically integrate the ODEs, exploiting its embedded event detection system to manage the firing of events. In particular, stochastic simulation is dealt with in the following way [40, 8]. Consider a stochastic event with rate $\lambda(t)$, depending on time via the continuously evolving variables of the system. We compute its cumulative rate $\Lambda\left(t_{0}, t\right)=\int_{t_{0}}^{t} \lambda(s) d s$ by coupling 
with the ODE system, the following equation for $\Lambda$ : $\frac{d \Lambda\left(t_{0}, t\right)}{d t}=\lambda(t)$, with $\Lambda\left(t_{0}, t_{0}\right)=0$. Then, we fire the stochastic transition as soon as $\Lambda\left(t_{0}, t\right)=-\ln (U)$, where $U \sim \operatorname{Unif}(0,1)$ is a uniformly distributed random number in $[0,1]$, sampled using the pseudo-number generator of MathCommons library. Notice that if the rate $\lambda(t)=\lambda$ is constant, then the firing time is $-\frac{1}{\lambda} \ln (U)$, and hence we have the standard Monte Carlo inversion method to simulate exponentially distributed random variables [40].

We conclude this section with some details of the language supported by the tool to model with HYPE. Each HYPE model consists of 6 sections. The \#definitions section contains the definition of system variables, parameters, expressions shorthands, user-defined functions (which replace influence types in the tool) and boolean predicates. The \#mappings section is devoted to the definition of influences (mapping them to variables) and events (specifying their name, guard, reset, and, for stochastic events, rate). In the tool, stochastic events can be guarded. This is rendered in HYPE using suitable discontinuous rate functions. The \#subcomponent section contains the description of subcomponents, which can be parameterised (with respect to variables and events) in order to reuse the same definition more than once. In particular, the user can assign more than one event to the same influence and the influence name is assigned to the whole subcomponent, in order to comply to the restrictions of well-defined HYPE models. The \#component section, instead, contains the (parametric) description of system components, including the uncontrolled system. The \#controller section contains the definition of sequential and compound controllers. Finally, the \#system section specifies the complete system by combining a controller and an uncontrolled system. The code for the example of Figure 1 is given in Figure 2. We would like to add additional parameterisation abilities to the software to support systems where we define many similar components. We address this point further in Section 6 .

\section{Opportunistic networks}

Since stochastic HYPE allows for the modelling of discrete quantities in a fluid manner, it is suitable for network modelling. Furthermore, it has stochastic aspects that model randomness, making it appropriate to model the disconnectedness that can happen in opportunistic networks. Networks are opportunistic when nodes can communicate even though there may never be a direct path between them. They use a store-carry-forward approach and decisions about routing are determined dynamically with policies based on the notion of getting a packet closer to its final destination [32]. Delays may occur but networks of this type can be deployed in environments where disconnectedness is possible but increased time for packet delivery is acceptable. The major challenges in such networks [32, 22, 35] include the following.

Disconnectedness: A direct path may occur very infrequently or never between any two nodes in the network.

High latency and low data rate: Due to disconnectedness, there can be significant delays for an individual packet, including those caused by queueing at an intermediate node. This obviously can result in low throughput.

Limited resources: Nodes are often battery-driven and hence need to conserve energy to lengthen their lifetimes. This means that the amount of storage space or strength and length of radio usage for communication are limited. Additionally, nodes may become permanently disabled due to a hostile environment.

Various protocols have been designed to mitigate the problems caused by these challenges and these protocols have specific objectives [35]. The main objective is to maximise the probability of a packet reaching its destination. Ideally, at the same time, both the delivery delay and the resource usage should 
be minimised. Storage capacity at nodes should be sufficient, both to cope with the inherent latency and in certain cases, to store copies of messages that could be lost.

There are different ways to categorise routing/forwarding protocols: deterministic versus stochastic [42], with or without infrastructure [32] or most commonly, flooding versus forwarding [22, 35]. In flooding protocols, packets are forwarded to many nodes. Variations include epidemic routing which is based on a model of disease transmission, where nodes that have "recovered" do not forward packets they have already seen [39]. Additional conditions can be added to epidemic routing to reduce resource usage. Other flooding approaches involve the estimation of the probability of delivery by a node, and this is used in deciding which nodes packets should be forwarded to. An example is PROPHET [30].

In forwarding protocols, a single copy of a packet moves through the network. Decisions about which node is the best node to move to can be done by location (how close the node is to the destination node), knowledge about the network provided by oracles [23] or other characteristics of the network that can be obtained by a node through interaction with other nodes. For example, the MaxProp protocol is based on historical data of path likelihood [9].

In the context of our case study, two wildlife monitoring projects are of interest: ZebraNet [25] and SWIM [36]. In the first case, zebra are fitted with collars and the data is collected by a mobile node on a vehicle that moves around the area. In second case, whales are tagged with sensors. In both cases, flooding can be used as the protocol. In flooding, whenever two animals are in sufficiently close proximity, they exchange data. In this way, assuming one animal comes close enough to the mobile nodes or base stations, there is sufficient proximity between animals, and no data buffers become full, all data will arrive at the base station. In the case of ZebraNet, the history-based protocol is also used. Here, each sensor keeps a value which gives an indication of when it last interacted with the mobile node. When deciding which neighbour sensor to send data to, the one with the highest value is chosen. This protocol has been shown to outperform the flooding protocol.

ZebraNet has similarities with carrier-based routing (which is classified by [32] as routing with mobile infrastructure). In these protocols [24, 43], particular mobile nodes which can be called carriers, supports, forwarders, mules or ferries, collect data from other, possibly stationary, nodes. In some protocols, only the ferries collect data and in others, non-ferry nodes exchange data as well. Our case study is based on the former.

\subsection{Our case study}

As an initial test for our modelling of opportunistic networks, we have chosen a relatively simple scenario. Since our fluid packet approach is most useful in cases where there are large amounts of data, we consider an example where video data is captured by stationary sensors. The idea is that they are motion activated with a low number of activations expected each day. Because these sensors are required to run off battery, it is not desirable for them to have powerful enough radio to share data over distance. Hence, a vehicular ferry moves around the area in which the video sensors are located and returns to a base station where the ferry delivers the data. This scenario could occur in a wildlife reserve or any scenario where video data is to be collected, but is not required in real-time. We do not assume that the primary purpose of the vehicle involved is to collect data from the nodes. It could be involved in supply delivery or game viewing, but we do assume some flexibility in routing as we consider later.

If we assume a fixed disk size for the video sensors, and no restriction on the amount of data that can be delivered to the base station, then the parameters of interest relate to the ferry and include buffer size, route taken and speed of movement. In the next section, we discuss how this can be modelled. 


\section{A framework for modelling opportunistic networks}

The basic element of our model is the network node. To be able to model opportunistic networks in the most general way, we assume that each node has a fixed buffer capacity, and that it can keep track of multiple streams of data. These streams could represent data with different priorities, data with different destinations or different types of data; or combinations of priority, destination and type.

A node should be able to accept input; offer output; discard data from the buffer, either to free up space by dropping current data or to remove stale data, and generate data. Moreover, it should be able to keep track of the total data it has dropped, input or generated. It should also be able to make decisions about what data to input (how much, which and from whom), output (how much, which and to whom) and drop (how much and which). In certain instances, it may also need to make decisions about what data to generate.

To model this node in stochastic HYPE, we require variables to capture the current buffer level for each stream, together with the lifetime input and generated data for each stream, and lifetime dropped data for each stream. This gives us variables Level $_{i, v}$, TotalIG $_{i, v}$ and TotalD $i, v$ for node $i$ and stream $v$. Clearly, the value of the variables for everything except buffer levels will not decrease.

Each node has subcomponents for input, output, generation, drop and removal (where the second last term refers to discarding current data and the last term to discarding stale data). It also has two further subcomponents to keep track of data input and data generated. We have the following HYPE components for each node $i$ and each stream $v$. Here, the symbol indicates synchronisation on all shared events.

$$
\text { Node }_{i, v} \stackrel{\text { def }}{=} \text { Input }_{i, v} \rightsquigarrow \text { Output }_{i, v} \rightsquigarrow \text { Generate }_{i, v} \rightsquigarrow \text { Remove }_{i, v} \rightsquigarrow \text { Drop }_{i, v} \rightsquigarrow \text { KeepI }_{i, v} \rightsquigarrow \text { Keep }_{i, v}
$$

The influences that appear in Input $_{i, v}$, Output $_{i, v}$, Generate $_{i, v}$ and Remove $_{i, v}$ are mapped to the variable Level $_{i, j}$, those in $\operatorname{KeepI}_{i, v}$ and $\operatorname{Keep}_{i, v}$ are mapped to the variable TotalIG $i, j$ and that in Drop $_{i, v}$ to the variable TotalD $i, j$.

Each node has a controller for the first five subcomponents. Controllers are not required for the last two subcomponents since the events for these subcomponents appear in the controllers for input and generation. Controllers are required for each stream as they may need to be treated separately, for example in the case of one stream being prioritised over another.

$$
\text { ConNode }_{i, v} \stackrel{\text { def }}{=} \operatorname{ConI}_{i, v} \operatorname{ConO}_{i, v} \rightsquigarrow \operatorname{ConG}_{i, v} \bowtie \operatorname{ConR}_{i, v} \operatorname{ConD}_{i, v}
$$

The example in Section 2 provides a very simple version of such a node which only has input and output capability. The controllers must deal with aspects such as full and empty buffers, as well as switching between different functions, for example switching between input on and input off.

The next important aspect to model is the interaction between nodes in the network. Each possible connection between two nodes (some nodes may never have the ability to connect) has a controller that synchronises on proximity and brings up the link, takes down the link at the end of proximity or due to any other condition that could cause the link to end, and then does some housekeeping. Hence, we define $\operatorname{ConL}_{i, j}$ the controller for the link between nodes $i$ and $j$.

Then for each stream of data, there is a controller that involves events that determine whether data exchange should happen for that stream and whether the link should be uni- or bidirectional. The directionality of the link depends both on the characteristics of the modelled system and the policies used. This controller also includes a housekeeping subcontroller that synchronises with the housekeeping of the controller of the link between the two nodes.

$$
\text { ConStream }_{i, j, v} \stackrel{\text { def }}{=} \text { ConUni }_{i, j, v} \text { ConUni }_{j, i, v} \text { CBi }_{i, j, v} \text { ConTidy }_{i, j, v}
$$


Finally, there are controllers which model the proximity of nodes. This is currently done abstractly, using a random variable to capture delays between connectedness. A recent paper describes the expected meeting time between nodes for various mobility models such as random direction, random waypoint and community-based (both for homogeneous and heterogeneous nodes) and suggest that these expected values can be used as rates to describe exponential distributions [37]. This allows us to model these specific types of mobility in this abstract fashion. However, as future work, we plan to develop more concrete models that describe movement in two-dimensional space, since HYPE can model this type of continuous behaviour in a straightforward way.

Hence, to construct an opportunistic network model in stochastic HYPE, we need to define a number of nodes based on our template, the appropriate connection controllers and proximity controllers, and most importantly, the policies and protocols that will be used in the network. Our longer-term goal is to develop a front end that will allow a user to specify nodes, connections, policies and protocols from which a HYPE model will be generated. This will provide a simulation tool for networks that should be faster than simulators that trace every packet, when the number of packets is large.

\subsection{Our case study}

For our specific case study, we used the basic node we have developed. Video sensor nodes require generation, discard and output capabilities; the ferry requires input and output, and the base station, input only. Possible links between nodes cover upload from sensors to the ferry and upload from the ferry to the base station. There are also two different proximity controllers: one allows the ferry to have a random route between nodes, and the other imposes a fixed route that is cyclically repeated.

In our case study, the policies are straightforward - unidirectional communication between a sensor and the ferry is set up only if the ferry is not currently communicating with another sensor. However, in a scenario with more than one ferry, it would be possible to implement a policy allowing a sensor node to choose which ferry to upload data to, making a decision based on certain ferry characteristics. Since we are dealing with a system where large amounts of data are generated, it makes sense to use this protocol rather than any other. In this scenario, it would be problematic to use flooding as the system is data bound, and hence an excessive transmission and storage of data cannot be recommended.

\subsection{Results}

The specifics of our case study are as follows: we assume that there are 10 video sensors and that the ferry only collects data during an 8 hour period, and we are interested both in what buffer size the ferry requires and how often there is contact between the ferry and a sensor (described by the mean-time-tocontact variable $M T C$ ), which is effectively the speed of the ferry.

Each video sensor has $250 \mathrm{MB}$ of disk space, and on average will record video three times a day for an average of 3 minutes each time; the video will require $10 \mathrm{Mb}$ for each minute. The upload speed from sensor to ferry is $1 \mathrm{MB} / \mathrm{s}$ and the upload speed from ferry to base station is $30 \mathrm{MB} / \mathrm{s}$.

We consider 4 different scenarios. For raer, the ferry only returns to base at the end of the 8 hour period and has a random route. In the case of raef, the return to base is also at the end but there is a fixed route. For $r t b r$ and $r t b f$, the ferry returns to base whenever it is full (incurring a penalty of extra distance to travel) and there are random and fixed routes, respectively. In the experiments to explore different values for mean time to contact, the ferry buffer size was set to $1000 \mathrm{MB}$. In the experiments to consider different ferry buffer size the mean time to contact was set to 15 minutes. Each simulation took around 6.5 seconds on a standard laptop. 


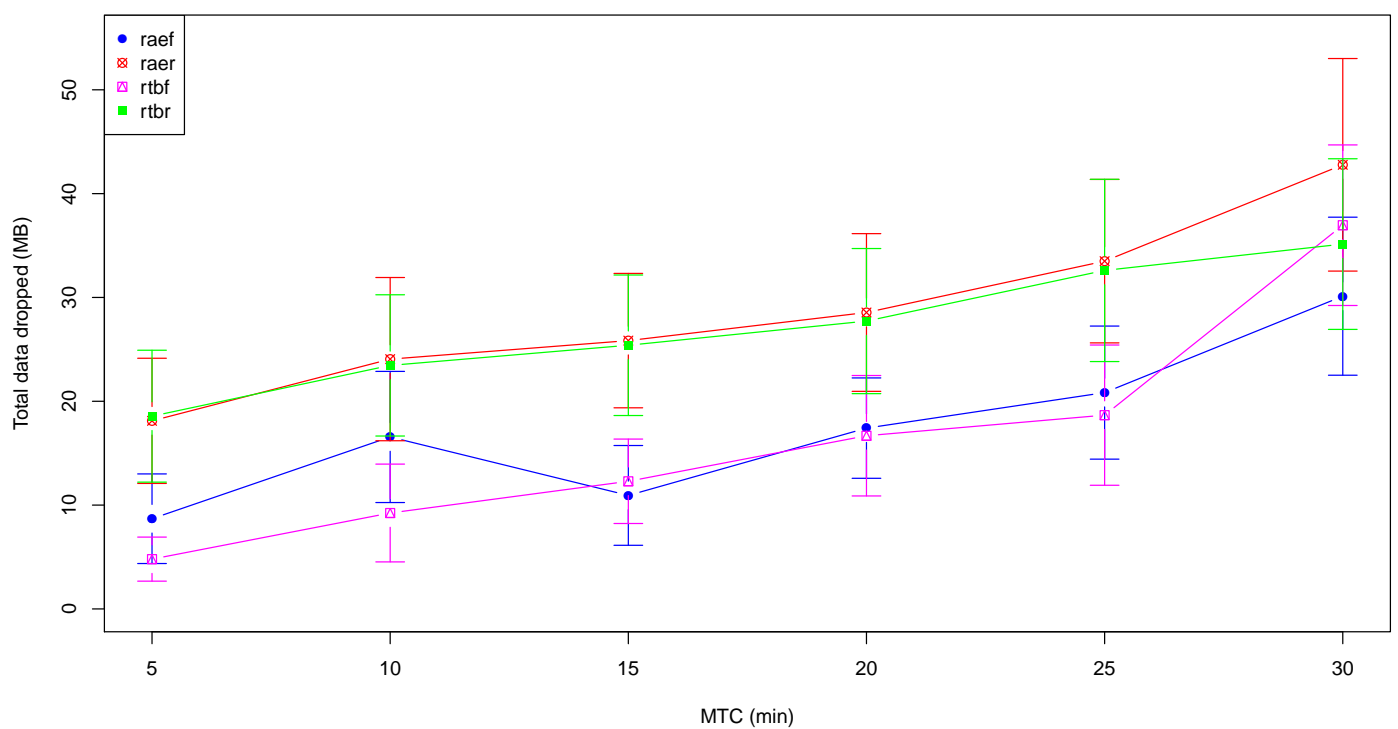

Figure 3: Average (200 simulation runs) data dropped for different mean time to contact (MTC) values. The bars delimit the $95 \%$ confidence interval. See the text for the description of the four different scenarios.

When we consider data dropped versus mean time to contact (see Figure 3), as mean time to contact increases (which means visits are less frequent) the amount of data dropped increases, which is what we would expect. In general, it can be seen that the fixed routes (raef amd $r t b f$ ) result in less data drop, which can be ascribed to fairness, in that each node get its turn whereas in the random case, it may not get a turn at all. Similarly, Figure 4 show that as frequency of visits decreases, the amount of data collected by the ferry decreases, and the fixed routes (raef amd $r t b f$ ) perform better in collecting more data.

For the ferry buffer size, we found that there appeared to be limited correlation between the different protocols and amount of data dropped, shown in Figure 5. By contrast, in Figure 6, the amount of data collected by the ferry, is understandably reduced for low buffer capacities in the case where the ferry does not return to base when full (raer and raef). At the higher buffer capacities, again the fixed routes out perform the random routes.

This case study illustrates how stochastic HYPE can be used to model these networks. It is relatively straightforward to add further nodes of all types, and hence model a larger system of the same type.

\section{Related work}

Other hybrid process algebras to describe hybrid systems include $A C P_{h s}^{s r t}$ [6], hybrid $\chi$ [4], $\phi$-calculus [34] and HyPA [10]. The aspect of HYPE that distinguishes it from these process algebra, is that in HYPE the ODEs emerge from the semantics and are not required to be specified monolithically in the syntax because of the use of individual flows in HYPE. Additionally, unlike the process algebras mentioned and hybrid automata, it is possible to combine two HYPE models where a variable can be shared between models since it is possible to combine all the influences that apply to this shared variable. A more detailed comparison between HYPE and other hybrid modelling formalisms can be found in [17, 16]. To the best 


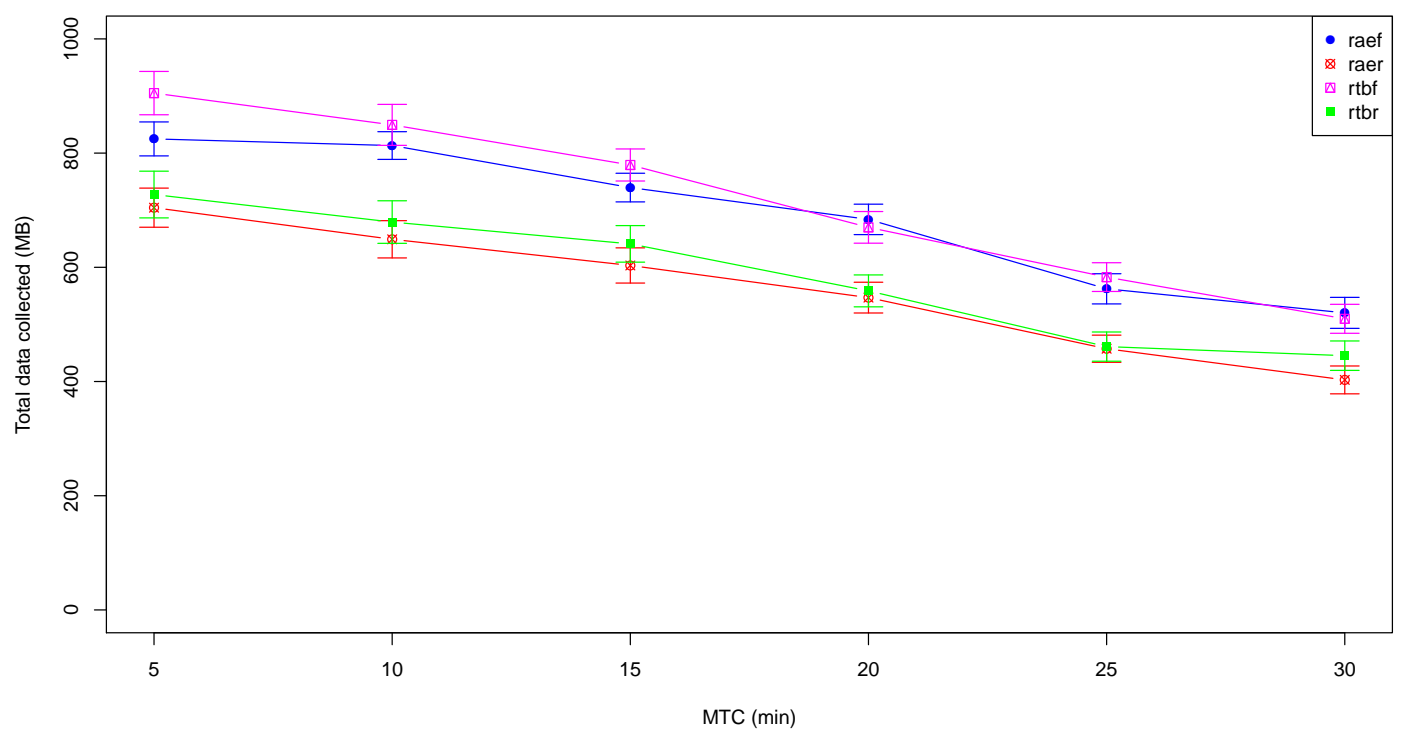

Figure 4: Average (200 simulation runs) data collected by ferry for different mean time to contact (MTC) values. The bars delimit the $95 \%$ confidence interval.

of our knowledge, no hybrid process algebra has previously been used to model networks using a fluid packet approach. Recently, an approach based on rewriting logic, Hybrid Interacting Maude, has been developed [14] but to date this research has focussed on thermal systems.

Other formal approaches to performance modelling of opportunistic networks have appeared in the literature in recent years. Much work has focussed on modelling the mobility patterns of nodes within the network, a feature that clearly has a strong impact on the performance that can be achieved. Examples include [41] and [37] in which the authors analyse the expected meeting time for various mobility models and bounds on delays. Other papers focus on the performance measures such as message delay and compare, as we do, the routing policies which may be applied. For example, Picu and Spyropoulos [33] use expensive Markov Chain Monte Carlo simulation to assess optimal relay selection for multicast communication in opportunistic networks, while [27] presents analytic bounds on message delivery capacity. Another example [31] considers the provisioning of a network in order to minimize the delay using an analytical model based on queueing theory. Their framework is more general than ours in the sense that services, rather than simply messages, are exchanged opportunistically between nodes and results as well as service requests are also exchanged. However, it should be possible to extend our modelling framework to encompass this richer scenario. Closest to our work in terms of formality is the work of Garetto and Gribaudo, but this presents a purely discrete model in terms of a state-labelled Markov chain which is subjected to probabilistic model checking [19] and is therefore limited in the size of system which can be considered.

Other simulators for opportunistic networks have been proposed, for example the ONE [26] and a virtual test platform [12]. These simulators work at the packet and message level and do not introduce a fluidisation of data flow. Additionally, examples studied quite often consider generation rates as low as a message an hour or a day. By using a fluid approach, we can model much higher generation rates.

Lastly, we mention other fluid approaches to modelling networks (necessarily incomplete due to 


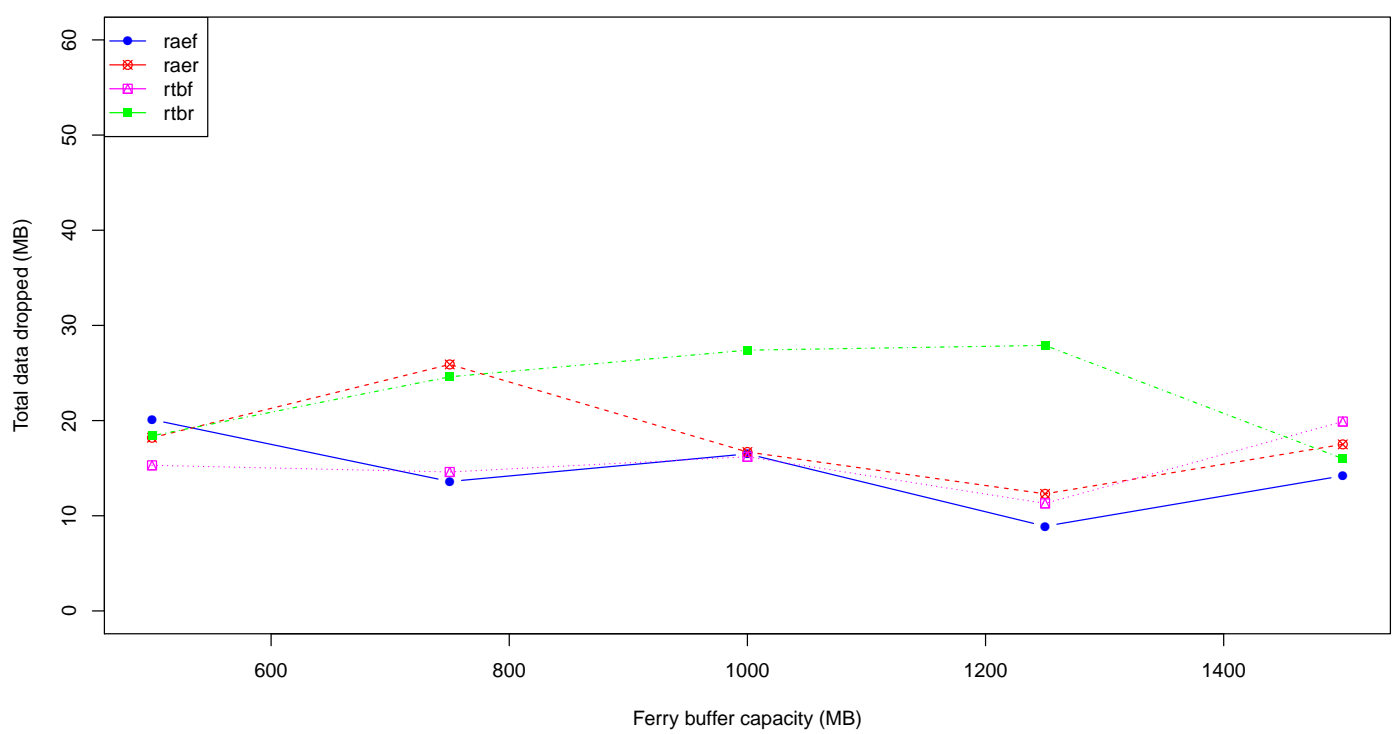

Figure 5: Average (100 simulation runs) data dropped for different ferry buffer sizes

space constraints). These include simulation [29], fluid stochastic Petri nets (FSPNs) [21, 38, 2, 15] and mean field approximations [5, 3]. These approaches, as far as we know, have not been applied to opportunistic networks and they do not offer the compositionality that a process algebra provides.

Petri net approaches include modelling a single cell of a wireless internet access system as a deterministic and stochastic Petri net; a generalised stochastic Petri net; and a FSPN [2]. The FSPN model is much faster to solve than the other two, and has good accuracy apart from a few specific scenarios. The FSPN model has a single fluid place to represent the buffer of the system. Another Petri net approach presents a FSPN model of client-server interaction in a peer-to-peer network [15] which is used to obtain distributions describing file transfer times. The single fluid place represents the download of a file. In both these examples, there is no flow between continuous places, and it is not clear how these models could be extended to model systems of multiple cells, or multiple clients and servers where continuous flows occur between different elements of the system, in contrast to our approach.

\section{Further research and conclusions}

In terms of future work, we plan to improve the software tool in at least two directions. First, we want to improve the modularisation of the input language, allowing the modeler to write parametric templates corresponding to generic system components like ferries, data stations, and connections. Secondly, we plan to implement in the hybrid simulation more clever management of the discrete variables denoting modes of the automaton, using data structures such as dependency graphs to reduce the amount of times each guard of a discrete transition is tested. Preliminary work on this (together with bytecode on-thefly compilation of mathematical expressions) has shown a thirty-fold increase in performance over the execution time given in Section 4.2. In addition, we plan to implement a multithread support to exploit multi-core processors to reduce simulation time.

As for the opportunistic networks, exploiting modularisation of HYPE code, we plan to define a 


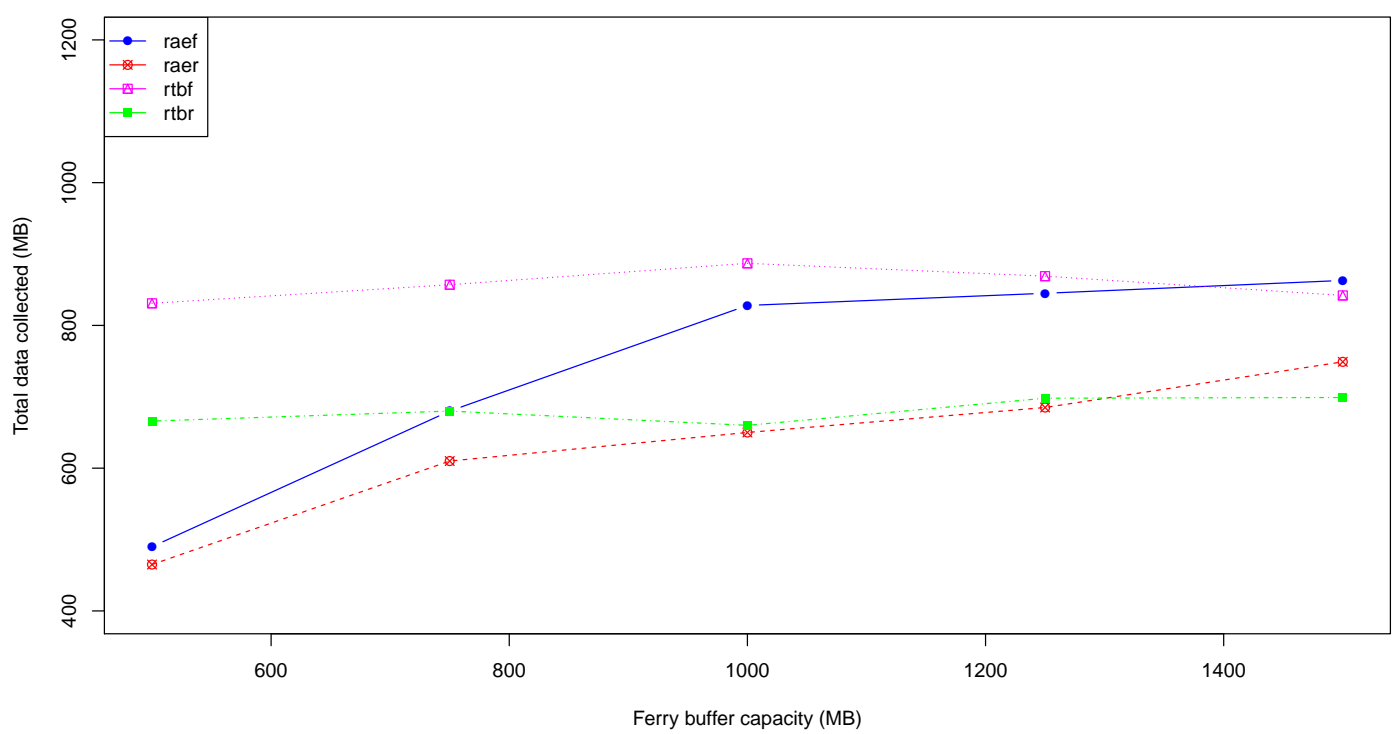

Figure 6: Average (100 simulation runs) data collected by ferry for different ferry buffer sizes

library of components and an higher level graphical interface to construct a model. As mentioned in Section 4, we wish to investigate the use of two-dimensional models of node movement. Furthermore, we plan to explore the use of HYPE bisimulations to reduce model size when possible. In certain cases, it may also be possible to reduce a sequence of events to one event while retaining the same behaviour with respect to simulation. As mentioned in Section 4.2 , it should be easy to increase the size of the networks to be modelled, and hence we will investigate how our approach scales and what limitations there might be.

As a general principle, hybrid simulation can be more efficient than discrete event simulation (without continuous flows) as long as the computation time for solving the ODEs is shorter than the computation time for simulating the events that the fluid approach removes. We wish to establish whether this principle holds in modelling opportunistic networks and what the trade-off is between efficiency and accuracy of the modelling. Currently, we have focussed on providing averages and standard deviations over a number of simulation runs. An alternative approach is to formally specify the properties we are interested in using a stochastic logic and then to apply statistical model checking.

To conclude, we have presented a general framework for constructing models of opportunistic networks using stochastic HYPE. We have illustrated this through a case study of a ferry that collects data from video nodes and delivers it to a base station. The results of our simulations of the model show, as we would expect, that fixed routes are likely to be more fair and have less data dropped and more data collected. Additionally, at low buffer sizes, the penalty of returning to the base is justified to avoid dropping data unnecessarily.

Acknowledgements: This research is supported by Royal Society International Joint Project JP090562.

\section{References}

[1] Apache Commons Math Java Library. Available at http://commons . apache.org/math/ 
[2] M. Ajmone Marsan, M. Gribaudo, M. Meo \& M. Sereno (2001): On Petri Net-based modeling paradigms for the performance analysis of wireless Internet accesses. In: 9th International Workshop on Petri Nets and Performance Models, pp. 19-28, doi:10.1109/PNPM.2001.953352.

[3] R. Bakhshi, L. Cloth, W. Fokkink \& B.R. Haverkort (2011): Mean-field framework for performance evaluation of push-pull gossip protocols. Performance Evaluation 68, pp. 157-179, doi:10.1016/j.peva.2010.08.025

[4] D.A. van Beek, K.L. Man, M.A. Reniers, J.E. Rooda \& R.R.H. Schiffelers (2006): Syntax and consistent equation semantics of hybrid $\chi$. Journal of Logic and Algebraic Programming 68, pp. 129-210, doi:10.1016/j.jlap.2005.10.005

[5] M. Benaïm \& J.-Y. Le Boudec (2008): A class of mean field interaction models for computer and communication systems. Performance Evaluation 65, pp. 823-838, doi:10.1016/j.peva.2008.03.005.

[6] J.A. Bergstra \& C.A. Middelburg (2005): Process algebra for hybrid systems. Theoretical Computer Science 335, pp. 215-280, doi:10.1016/j.tcs.2004.04.019.

[7] L. Bortolussi, V. Galpin \& J. Hillston (2011): HYPE with stochastic events. In: QAPL 2011, EPTCS 57, pp. 120-133, doi:10.4204/EPTCS.57.9

[8] L. Bortolussi \& A. Policriti (2009): Hybrid semantics of stochastic programs with dynamic reconfiguration. In: COMPMOD 2009, EPTCS 6, pp. 63-76, doi:10.4204/EPTCS.6.5.

[9] J. Burgess, B. Gallagher, D. Jensen \& B.N. Levine (2006): MaxProp: Routing for vehicle-based disruptiontolerant networks. In: INFOCOM 2006, pp. 1-11, doi:10.1109/INFOCOM.2006.228.

[10] P.J.L. Cuijpers \& M.A. Reniers (2005): Hybrid process algebra. Journal of Logic and Algebraic Programming 62, pp. 191-245, doi $10.1016 /$ j.jlap.2004.02.001

[11] M.H.A. Davis (1993): Markov Models and Optimization. Chapman \& Hall.

[12] N.A. Deepak, R. Thareja \& N.A. Nikhil (2008): Performance analysis and evaluation of delay-tolerant network bundling protocol on a scalable virtual network test platform. In: IET International Conference on Wireless, Mobile and Multimedia Networks, 2008, pp. 52 -55, doi:10.1049/cp:20080143.

[13] M.B. Elowitz \& S. Leibler (2000): A synthetic oscillatory network of transcriptional regulators. Nature 403, pp. 335-338, doi: $10.1038 / 35002125$

[14] M. Fadlisyah, P.C. Ölveczky \& E. Ábrahám (2011): Object-oriented formal modeling and analysis of interacting hybrid systems in HI-Maude. In: SEFM 2011, LNCS 7041, pp. 415-430, doi:10.1007/978-3-64224690-6_29.

[15] R. Gaeta, M. Gribaudo, D. Manini \& M. Sereno (2005): Fluid stochastic Petri nets for computing transfer time distributions in peer-to-peer file sharing applications. ENTCS 128, pp. 79-99, doi $10.1016 /$ j.entcs.2005.01.014

[16] V. Galpin, L. Bortolussi \& J. Hillston: HYPE: Hybrid modelling by composition of flows, doi $10.1007 / \mathrm{s} 00165-011-0189-0$. Formal Aspects of Computing, to appear.

[17] V. Galpin, L. Bortolussi \& J. Hillston (2009): HYPE: a process algebra for compositional flows and emergent behaviour. In: CONCUR 2009, LNCS 5710, pp. 305-320, doi:10.1007/978-3-642-04081-8_21.

[18] V. Galpin, J. Hillston \& L. Bortolussi (2008): HYPE applied to the modelling of hybrid biological systems. ENTCS 218, pp. 33-51, doi $10.1016 /$ j.entcs.2008.10.004

[19] M. Garetto \& M. Gribaudo (2006): Performance analysis of delay tolerant networks with model checking techniques. In: QEST 2006, pp. 73-82, doi 10.1109/QEST.2006.42

[20] T.A. Henzinger \& P.-H. Ho (1995): HYTECH: The Cornell HYbrid TECHnology Tool. In: Hybrid Systems II, LNCS 999, pp. 265-293, doi:10.1007/3-540-60472-3_14.

[21] G. Horton, V.G. Kulkarni, D.M. Nicol \& K.S. Trivedi (1998): Fluid stochastic Petri nets: Theory, applications, and solution techniques. European Journal of Operational Research 105, pp. 184 - 201, doi: $10.1016 / \mathrm{S} 0377-2217(97) 00028-3$

[22] C.-M. Huang, K.-C. Lan \& C.-Z. Tsai (2008): A survey of opportunistic networks. In: AINA 2008, pp. 1672-1677, doi:10.1109/WAINA.2008.292 
[23] S. Jain, K.R. Fall \& R.K. Patra (2004): Routing in a delay tolerant network. In: ACM SIGCOMM 2004, pp. 145-158, doi: $10.1145 / 1015467.1015484$

[24] S. Jain, R. Shah, W. Brunette, G. Borriello \& S. Roy (2006): Exploiting mobility for energy efficient data collection in wireless sensor networks. MONET 11, pp. 327-339, doi 10.1007/s11036-006-5186-9.

[25] P. Juang, H. Oki, Y. Wang, M. Martonosi, L.-S. Peh \& Daniel Rubenstein (2002): Energy-efficient computing for wildlife tracking: design tradeoffs and early experiences with ZebraNet. ACM SIGPLAN Notices 37, pp. 96-107, doi $10.1145 / 605397.605408$

[26] A. Keränen, T. Kärkkäinen \& J. Ott (2010): Simulating mobility and DTNs with the ONE. Journal of Communications 5, pp. 92-105, doi $10.4304 / \mathrm{jcm} .5 .2 .92-105$.

[27] G.Y. Keung, B. Li \& Q. Zhang (2011): Message delivery capacity in delay-constrained mobile sensor networks: Bounds and realization. IEEE Transactions on Wireless Communications 10, pp. 1552-1559, doi $10.1109 /$ TWC.2011.030911.100827

[28] U. Khadim (2006): A comparative study of process algebras for hybrid systems. Report CSR 06-23, Technische Universiteit Eindhoven. http://alexandria.tue.nl/extra1/wskrap/publichtml/200623.pdf

[29] C. Kiddle, R. Simmonds, C. Williamson \& B. Unger (2003): Hybrid packet/fluid flow network simulation. In: PADS 2003, pp. 143 - 152, doi 10.1109/PADS.2003.1207430

[30] A. Lindgren, A. Doria \& O. Schelén (2003): Probabilistic routing in intermittently connected networks. Mobile Computing and Communications Review 7, pp. 19-20, doi:10.1145/961268.961272

[31] A. Passarella, M. Kumar, M. Conti \& E. Borgia (2011): Minimum-delay service provisioning in opportunistic networks. IEEE Transactions on Parallel and Distributed Systems 22, pp. 1267-1275, doi $10.1109 /$ TPDS.2010.153.

[32] L. Pelusi, A. Passarella \& M. Conti (2006): Opportunistic networking: data forwarding in disconnected mobile ad hoc networks. IEEE Communications Magazine 44, pp. 134-141, doi:10.1109/MCOM.2006.248176.

[33] A. Picu \& T. Spyropoulos (2010): Distributed stochastic optimization in opportunistic networks: the case of optimal relay selection. In: CHANTS '10, pp. 21-28, doi:10.1145/1859934.1859939

[34] W.C. Rounds \& H. Song (2003): The $\Phi$-Calculus: A language for distributed control of reconfigurable embedded systems. In: HSCC 2003, LNCS 2623, pp. 435-449, doi 10.1007/3-540-36580-X_32.

[35] J. Shen, S. Moh \& I. Chen (2008): Routing protocols in delay tolerant networks: A comparative survey. In: 23rd International Technical Conference on Circuits/Systems, Computers and Communications (ITC-CSCC 2008), pp. 1577-1580.

[36] T. Small \& Z.J. Haas (2003): The shared wireless infostation model: a new ad hoc networking paradigm (or where there is a whale, there is a way). In: MobiHoc 2003, pp. 233-244, doi:10.1145/778415.778443.

[37] T. Spyropoulos, K. Psounis \& C.S. Raghavendra (2006): Performance analysis of mobility-assisted routing. In: MobiHoc 2006, pp. 49-60, doi:10.1145/1132905.1132912

[38] B. Tuffin, D.S. Chen \& K.S. Trivedi (2001): Comparison of hybrid systems and fluid stochastic Petri nets. Discrete Event Dynamic Systems: Theory and Applications 11, pp. 77-95, doi:10.1023/A:1008387132533.

[39] A. Vahdat \& D. Becker (2000): Epidemic routing for partially connected ad hoc networks. Technical Report CS-2000-06, Duke University. Available at issg.cs.duke.edu/epidemic/epidemic.pdf.

[40] D. Wilkinson (2011): Stochastic Modelling for Systems Biology. Chapman \& Hall/CRC.

[41] K. Xu, P. Hui, V.O.K. Li, J. Crowcroft, V. Latora \& P. Lio (2009): Impact of altruism on opportunistic communications. In: First International Conference on Ubiquitous and Future Networks, ICUFN'09, pp. 153-158, doi:10.1109/ICUFN.2009.5174303.

[42] Z. Zhang (2006): Routing in intermittently connected mobile ad hoc networks and delay tolerant networks: Overview and challenges. IEEE Communications Surveys and Tutorials 8, pp. 24-37, doi $10.1109 /$ COMST.2006.323440

[43] W. Zhao, M.H. Ammar \& E.W. Zegura (2004): A message ferrying approach for data delivery in sparse mobile ad hoc networks. In: MobiHoc 2004, pp. 187-198, doi:10.1145/989459.989483 